\title{
Simulator Based on a Simple Biped System
}

\author{
Sandra Cuatlaxahue Formacio and Pablo Sánchez-Sánchez \\ Benemérita Universidad Autónoma de Puebla, \\ Facultad de Ciencias de la Electrónica \\ robotics group oocelo \\ Puebla, México \\ cformaciosd@gmail.com \\ lepable@ece.buap.mx
}

\begin{abstract}
This study shows the mathematical modeling and the development of the simulator of a simple biped system in Matlab ${ }^{\circledR}$. We used specific libraries of Matlab $^{(0)}$ that allowed us to simulate mechanical systems. In order to design the 3D model, we used SolidWorks ${ }^{\circledR}$. The biped system is based on the structure of lower limb exoskeleton which is used in medical rehabilitation. We present the dynamic model calculation of the biped system through Euler-Lagrange method, and the stability analysis using Lyapunov theory. We present the implementation of a tracking control structure using a trajectory defined by fifth-order polynomials. The main consideration in this work is that the system is free of interaction with the environment, i.e., we discussed the ideal case.
\end{abstract}

Keywords: Simulator, dynamic model, biped system, lower limbs, fifthorder polynomial

\section{Introduction}

A computer simulation is an attempt to model a real-life or hypothetical situation on a computer so that it can be studied to see how the system works. By changing variables in the simulation, predictions may be made about the behaviour of the system. Simulator is a program that simulates specific conditions or the characteristics of a real process or machine for the purposes of research or study. Currently, simulators are being increasingly used in different areas of knowledge. Simulators in engineering are used in the design of complex systems. The results obtained in the analysis of data allow us to understand and improve the performance of the system[1]. An approach in robotics is the autonomous or semi-autonomous system design with ability to interact with its environment. Since complexity of robot control is increasing, simulators are becoming essential tools to understand the behavior of the system. The simulation allow us to enhance the design of the system and eliminate mechanical failures, before building the prototype $[2,3]$. The study of the bipedal robots has been around for more than three decades and there are still problems to be solved. Accordingly, this paper is focused on the development of a simulator based on a simple biped system with the purpose to study the major physical characteristics. 


\section{Modeling of the System}

The fixed frame is located at the base of the hip. The system is divided into 2 subsystems, right leg $\{S P D\}$ and left leg $\{S P I\}$. The first step is to obtain the forward kinematics of the system. The forward kinematics consists in obtaining the spatial location of the links with respect to a fixed coordinate system [4]. In order to analyze the system we requires a motion diagram, Fig. 1(a). The diagram is used to obtain the forward kinematics.

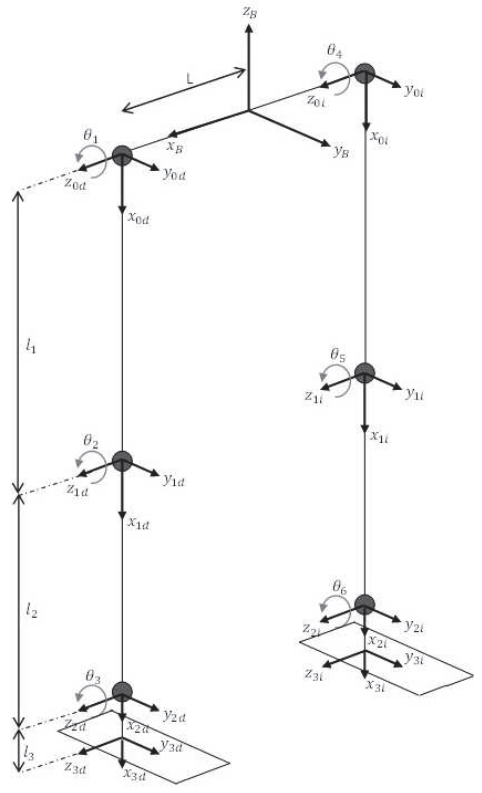

(a) coordinate system

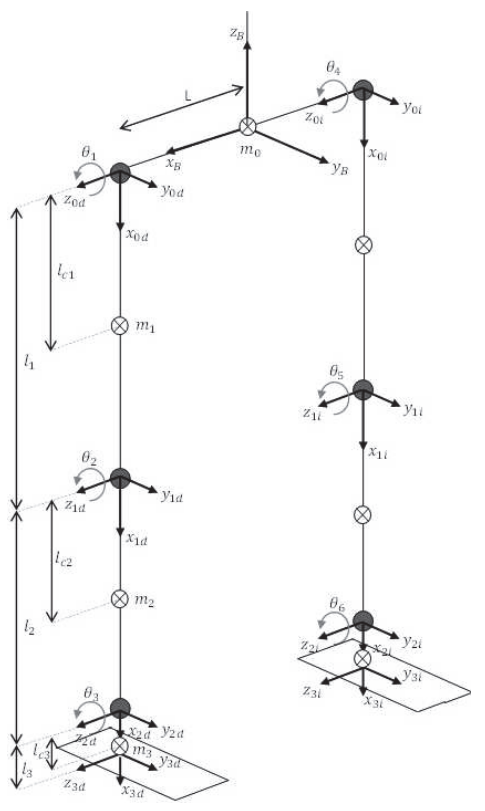

(b) Mass distribution

Fig. 1. Biped system frame

The forward kinematics is the first and most important step for the application of Euler-Lagrange method. The diagram of mass distribution is used for the analysis of velocity for each leg, Fig. 1(b).

\subsection{Forward Kinematics Model}

In order to obtain the homogeneous transformation matrices (spatial location of each link) we used the Denavit-Hartenberg algorithm [5]. The location of each link is obtained with respect to the fixed reference system located at the base of the system, Fig. $\mathbf{1}(\mathbf{a})$. 
The transformation matrices for $\{S P D\}$ are the following:

$$
\begin{aligned}
{ }^{B} A_{0 d}= & {\left[\begin{array}{cccc}
0 & 0 & 1 & L \\
0 & 1 & 0 & 0 \\
-1 & 0 & 0 & 0 \\
0 & 0 & 0 & 1
\end{array}\right] } \\
{ }^{B} A_{1 d}= & {\left[\begin{array}{cccc}
0 & 0 & 1 & L \\
\sin \left(\theta_{1}\right) & \cos \left(\theta_{1}\right) & 0 & l_{1} \sin \left(\theta_{1}\right) \\
-\cos \left(\theta_{1}\right) & \sin \left(\theta_{1}\right) & 0 & -l_{1} \cos \left(\theta_{1}\right) \\
0 & 0 & 0 & 1
\end{array}\right] } \\
{ }^{B} A_{2 d}= & {\left[\begin{array}{cccc}
0 & 0 & 1 & L \\
S \theta_{12} & C \theta_{12} & 0 & l_{2} S \theta_{12}+l_{1} S \theta_{1} \\
-C \theta_{12} & S \theta_{12} & 0 & -l_{2} C \theta_{12}-l_{1} C \theta_{1} \\
0 & 0 & 0 & 1 \\
0 & 0 & 1 & L \\
S \theta_{123} & C \theta_{123} & 0 & l_{3} S \theta_{123}+l_{2} S \theta_{12}+l_{1} S \theta_{1} \\
-C \theta_{123} & S \theta_{123} & 0 & -l_{3} C \theta_{123}-l_{2} C \theta_{12}-l_{1} C \theta_{1} \\
0 & 0 & 0 & 1
\end{array}\right] } \\
{ }^{B} A_{3 d}= & {\left[\begin{array}{cccc}
1
\end{array}\right] }
\end{aligned}
$$

where $S \theta_{1}=\sin \left(\theta_{1}\right), C \theta_{1}=\cos \left(\theta_{1}\right), S \theta_{12}=\sin \left(\theta_{1}+\theta_{2}\right), C \theta_{12}=\cos \left(\theta_{1}+\theta_{2}\right)$, $C \theta_{123}=\cos \left(\theta_{1}+\theta_{2}+\theta_{3}\right)$ y $S \theta_{123}=\sin \left(\theta_{1}+\theta_{2}+\theta_{3}\right)$.

$\{S P I\}$ has the same equations that $\{S P D\}$, the only difference is the position of the $x$-axis since $L$ is now $-L$ and it is evaluated with the corresponding angles, as seen in Fig. 1(a).

\subsection{Dynamic Model}

In the design of robots, control algorithms and simulators is important to consider the equations of motion [5]. We introduce the Euler-Lagrange equations, which describe the evolution of a mechanical system. The equation of motion is the following:

$$
\frac{d}{d t}\left[\frac{\partial \mathcal{L}(\theta, \dot{\theta})}{\partial \dot{\theta}}\right]-\frac{\partial \mathcal{L}(\theta, \dot{\theta})}{\partial \theta}=\tau
$$

where $\theta \in \mathbb{R}^{n}$ is the vector of generalized joint coordinates, $\tau \in \mathbb{R}^{n}$ is the vector of torques that act in the joints, and $\mathcal{L}(\theta, \dot{\theta})$ is know as Lagrangian, which is the difference between the kinetic and potential energy:

$$
\mathcal{L}(\theta, \dot{\theta})=\mathcal{K}(\theta, \dot{\theta})-\mathcal{U}(\theta)
$$

Considering that the biped system had no interaction with the environment, we can identify the input variables as the applied torques and the output variables as the positions [5]. 
By solving the Euler-Lagrange equation of motion described in (5) we obtain the dynamic model. The dynamic model is defined as:

$$
M(\theta) \ddot{\theta}+C(\theta, \dot{\theta}) \dot{\theta}+g(\theta)=\tau
$$

where $\theta \in \mathbb{R}^{n}$ is the vector of generalized joint coordinates, $M(\theta) \in \mathbb{R}^{n \times n}$ is the symmetric positive definite inertia matrix, $C(\theta, \dot{\theta}) \in \mathbb{R}^{n \times n}$ is the matrix of centripetal and Coriolis torques, $g(\theta) \in \mathbb{R}^{n}$ is the vector of gravitational torques, $\tau \in \mathbb{R}^{n}$ is the vector of torques that act in the joints. Assuming for simplicity that all robots have revolute joints, we can set the following properties [5]:

Property $1 M(\theta)$ satisfies $\lambda_{m}\|x\|^{2} \leq x^{T} M x \leq \lambda_{M}\|x\|^{2} \forall \theta, x \in \mathbb{R}^{n_{i}}$ where $\lambda_{m} \triangleq \min _{\forall \theta \in \mathbb{R}^{n_{i}}} \lambda_{\min }(M), \lambda_{M} \triangleq \max _{\forall \theta \in \mathbb{R}^{n_{i}}} \lambda_{\max }(M)$, and $0<\lambda_{m} \leq \lambda_{M}<\infty$.

Property $2 M(\theta)$ satisfies $0<\lambda_{m} \leq\|M(\theta)\| \leq \lambda_{M}<\infty$.

Property $3 M^{-1}$ satisfies $\sigma_{m}\|x\|^{2} \leq x^{T} M^{-1} x \leq \sigma_{M}\|x\|^{2} \forall \theta, x \in \mathbb{R}^{n_{i}}$, $\sigma_{m} \triangleq \min _{\forall \theta \in \mathbb{R}^{n_{i}}} \lambda_{\min }\left(M^{-1}\right)$, and $0<\sigma_{m} \leq \sigma_{M}<\infty$. $M^{-1}$ satisfies $0<\sigma_{m} \leq$ $\left\|M^{-1}\right\| \leq \sigma_{M}<\infty$.

Property $4 M(\theta)$ has the following relationship with the kinematic energy $\mathcal{K}(\theta, \dot{\theta})=\frac{1}{2} \dot{\theta}^{T} M(\theta) \dot{\theta}$

Property 5 The vector $C(\theta, x)$ y satisfies $C(\theta, x) y=C(\theta, y) x \forall x, y \in \mathbb{R}^{n}$.

Property 6 With the proper definition of $C(\theta, \dot{\theta})$, the matrix $\frac{1}{2} \dot{\theta}^{T}[\dot{M}(\theta)-2 C(\theta, \dot{\theta})] \dot{\theta} \equiv 0$ is skew-symmetric.

Property $7 C^{T}(\theta, \dot{\theta})=\frac{1}{2} \frac{\partial}{\partial \theta}\left[\dot{\theta}^{T} M(\theta) \dot{\theta}\right]$. 
The dynamic model for $\{S P D\}$ is defined as:

$$
\left[\begin{array}{lll}
m_{11} & m_{12} & m_{13} \\
m_{12} & m_{22} & m_{23} \\
m_{13} & m_{23} & m_{33}
\end{array}\right]\left[\begin{array}{l}
\ddot{\theta}_{1} \\
\ddot{\theta}_{2} \\
\ddot{\theta}_{3}
\end{array}\right]+\left[\begin{array}{lll}
c_{11} & c_{12} & c_{13} \\
c_{21} & c_{22} & c_{23} \\
c_{31} & c_{32} & c_{33}
\end{array}\right]\left[\begin{array}{c}
\dot{\theta}_{1} \\
\dot{\theta}_{2} \\
\dot{\theta}_{3}
\end{array}\right]+\left[\begin{array}{l}
g_{1} \\
g_{2} \\
g_{3}
\end{array}\right]=\left[\begin{array}{c}
\tau_{1} \\
\tau_{2} \\
\tau_{3}
\end{array}\right]
$$

where

$$
\begin{aligned}
& m_{11}=m_{1} l_{c 1}^{2}+m_{2} l_{1}^{2}+m_{3} l_{1}^{2}+m_{2} l_{c 2}^{2}+m_{3} l_{2}^{2}+2\left(m_{2} l_{c 2} l_{1}+m_{3} l_{2} l_{1}\right) \cos \left(\theta_{2}\right) \\
& +m_{3} l_{c 3}^{2}+2 m_{3} l_{c 3} l_{2} \cos \left(\theta_{3}\right)+2 m_{3} l_{c 2} l_{1} \cos \left(\theta_{2}+\theta_{3}\right)+I_{1}+I_{2}+I_{3} \\
& m_{12}=m_{2} l_{c 2}+m_{3} l_{2}^{2}+\left(m_{2} l_{c 2} l_{1}+m_{3} l_{2} l_{1}\right) \cos \left(\theta_{2}\right)+2 m_{3} l_{c 3} l_{2} \cos \left(\theta_{3}\right) \\
& +m_{3} l_{c 3} l_{1} \cos \left(\theta_{2}+\theta_{3}\right)+m_{3} l_{c 3}^{2}+I_{2}+I_{3} \\
& m_{13}=m_{3} l_{c 3}^{2}+m_{3} l_{c 3} l_{2} \cos \left(\theta_{3}\right)+m_{3} l_{c 3} l_{1} \cos \left(\theta_{2}+\theta_{3}\right)+I_{3} \\
& m_{22}=m_{2} l_{c 2}^{2}+m_{3} l_{2}^{2}+m_{3} l_{c 3}^{2}+2 m_{3} l_{c 3} l_{2} \cos \left(\theta_{3}\right)+I_{2}+I_{3} \\
& m_{23}=m_{3} l_{c 3}^{2}+m_{3} l_{c 3} l_{2} \cos \left(\theta_{3}\right)+I_{3} \\
& m_{33}=m_{3} l_{c 3}^{2}+I_{3} \\
& c_{11}=-2\left(m_{2} l_{c 2} l_{1}+m_{3} l_{2} l_{1}\right) \sin \left(\theta_{2}\right) \dot{\theta}_{2}-2 m_{3} l_{c 3} l_{2} \sin \left(\theta_{3}\right) \dot{\theta}_{3} \\
& +2 m_{3} l_{c 3} l_{1} \sin \left(\theta_{2}+\theta_{3}\right)\left(\dot{\theta}_{2}+\dot{\theta}_{3}\right) \\
& c_{12}=-2 m_{3} l_{c 3} l_{2} \sin \left(\theta_{3}\right) \dot{\theta}_{3}-m_{3} l_{c 3} l_{1} \sin \left(\theta_{2}+\theta_{3}\right)\left(\dot{\theta}_{2}+\dot{\theta}_{3}\right) \\
& c_{13}=-m_{3} l_{c 3} l_{2} \sin \left(\theta_{3}\right) \dot{\theta}_{3}-m_{3} l_{c 3} l_{1} \sin \left(\theta_{2}+\theta_{3}\right)\left(\dot{\theta}_{2}+\dot{\theta}_{3}\right) \\
& c_{21}=\left(m_{2} l_{c 2}+m_{3} l_{2}\right) l_{1} \sin \left(\theta_{2}\right) \dot{\theta}_{1}+m_{3} l_{c 3} l_{1} \sin \left(\theta_{2}+\theta_{3}\right) \dot{\theta}_{1} \\
& -2 m_{3} l_{c 3} l_{2} \sin \left(\theta_{3}\right) \dot{\theta}_{3} \\
& c_{22}=-2 m_{3} l_{c 3} l_{2} \sin \left(\theta_{3}\right) \dot{\theta}_{3} \\
& c_{23}=-m_{3} l_{c 3} l_{2} \sin \left(\theta_{3}\right) \dot{\theta}_{3} \\
& c_{31}=m_{3} l_{c 3} l_{2} \sin \left(\theta_{3}\right) \dot{\theta}_{1}+2 m_{3} l_{c 3} l_{2} \sin \left(\theta_{3}\right) \dot{\theta}_{2}+m_{3} l_{c 3} l_{1} \sin \left(\theta_{2}+\theta_{3}\right) \dot{\theta}_{1} \\
& c_{32}=m_{3} l_{c 3} l_{2} \sin \left(\theta_{3}\right) \dot{\theta}_{2} \\
& c_{33}=0 \\
& g_{1}=\left[m_{1} l_{c 1}+m_{2} l_{1}+m_{3} l_{1}\right] \sin \left(\theta_{1}\right)+\left[m_{2} l_{c 2}+m_{3} l_{2}\right] \sin \left(\theta_{1}+\theta_{2}\right) \\
& +m_{3} l_{c 3} \sin \left(\theta_{1}+\theta_{2}+\theta_{3}\right) \\
& g_{2}=\left[m_{2} l_{c 2}+m_{3} l_{2}\right] g \sin \left(\theta_{1}+\theta_{2}\right)+m_{3} l_{c 3} g \sin \left(\theta_{1}+\theta_{2}+\theta_{3}\right) \\
& g_{3}=m_{3} l_{c 3} g \sin \left(\theta_{1}+\theta_{2}+\theta_{3}\right)
\end{aligned}
$$

Owing to similarity of the reference system definition for both subsystems, $\{S P I\}$ has the same equations as $\{S P D\}$, except that it was evaluated with $\theta_{4}, \theta_{5}$ and $\theta_{6}$. 


\subsection{Control Structure}

In order to move the joints from an initial point to desired point (position control) or move the joints along a defined trajectory (control of movement), we applied a control structure, which allows the system to perform the assigned task [5]. The control structure is defined as follows:

$$
\tau=K_{p} \tilde{\theta}+K_{v} \dot{\tilde{\theta}}+g(\theta)
$$

where $K_{p}, K_{v} \in \mathbb{R}^{n \times n}$ are the proportional and derivative gains, respectively. The control structure described in (27) use the positions, velocity and gravitational torque information [5]. Note that the system is controlled in closed-loop as shown in Fig. 2.

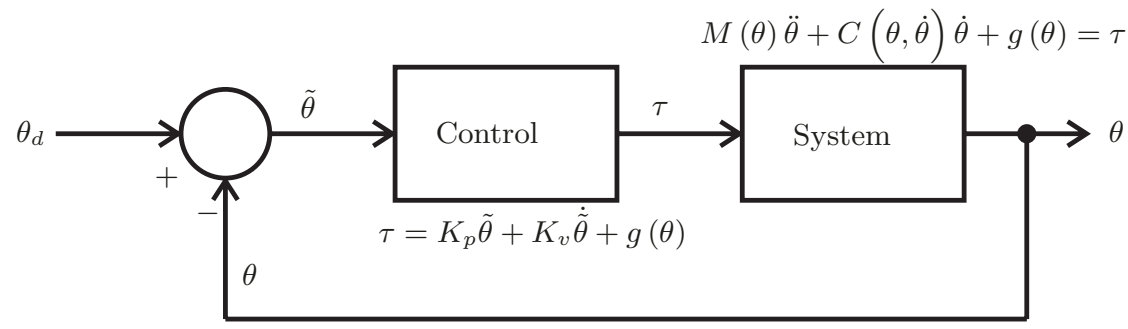

Fig. 2. System with control loop (inputs and outputs)

Demonstration of Stability. In order to demonstrate the stability of the system and the control structure, we use the Lyapunov theory. The first step is to design the matrices $K_{p}$ and $K_{v}$ such that the position error $\tilde{\theta}$ asymptotically vanishes, i. e. $\lim _{t \rightarrow \infty} \tilde{\theta}(t)=0 \in \mathbb{R}^{n}[5]$.

The closed-loop system equation obtained by combining the dynamic model described in (7), and control structure in (27), can be written as:

$$
\frac{d}{d t}\left[\begin{array}{c}
\tilde{\theta} \\
\dot{\theta}
\end{array}\right]=\left[\begin{array}{c}
-\dot{\theta} \\
M(\theta)^{-1}\left[K_{p} \tilde{\theta}-K_{v} \dot{\theta}-C(\theta, \dot{\theta}) \dot{\theta}\right]
\end{array}\right]
$$

(28) is known as closed-loop equation, which is an autonomous differential equation. Therefore, the origin of the state space is its unique equilibrium point [5]. In order to carry out the stability analysis of (28), the following Lyapunov function candidate based on the energy shaping methodology was proposed [5]:

$$
V(\dot{\theta}, \tilde{\theta})=\frac{\dot{\theta}^{T} M(\theta) \dot{\theta}}{2}+\frac{\tilde{\theta}^{T} K_{p} \tilde{\theta}}{2}
$$

Since $M(\theta)$ is a positive definite matrix the first term of $V(\dot{\theta}, \tilde{\theta})$ is a positive definite function with respect to $\dot{\theta}$. The second one term is a positive definite 
function with respect to position error $\tilde{\theta}$, because $K_{p}$ is a positive definite matrix. Therefore $V(\dot{\theta}, \tilde{\theta})$ is a globally positive definite and radially unbounded function[5]. The derivative of Lyapunov function candidate (29) along the trajectories of the closed-loop (28) is:

$$
\dot{V}(\dot{\theta}, \tilde{\theta})=\dot{\theta}^{T} M(\theta) \ddot{\theta}+\frac{\dot{\theta}^{T} \dot{M}(\theta) \dot{\theta}}{2}+\tilde{\theta}^{T} K_{p} \dot{\tilde{\theta}}
$$

and after some algebra and using the Property 6 it can be written as:

$$
\dot{V}(\dot{\theta}, \tilde{\theta})=-\dot{\theta}^{T} K_{v} \dot{\theta} \leq 0,
$$

which is a globally negative semidefinite function. Therefore, we concluded stability of the equilibrium point. In order to prove asymptotic stability, we applied the LaSalle invariance principle

$$
\dot{V}(\dot{\theta}, \tilde{\theta})<0
$$

In the region

$$
\Omega=\left\{\left[\begin{array}{c}
\tilde{\theta} \\
\dot{\theta}
\end{array}\right] \in \mathbb{R}^{n}: V(\tilde{\theta}, \dot{\theta})=0\right\}
$$

the unique invariant is $\left[\begin{array}{cc}\tilde{\theta}^{T} & \dot{\theta}^{T}\end{array}\right]^{T}=0 \in \mathbb{R}^{2 n}[5]$.

\subsection{Planning of Trajectory}

The biped system consisting of hip, knee and ankle must perform the movements of the human walking. In order to define the desired trajectory, we used the sagittal-plane motion of the lower extremity, Fig. 3 [6].

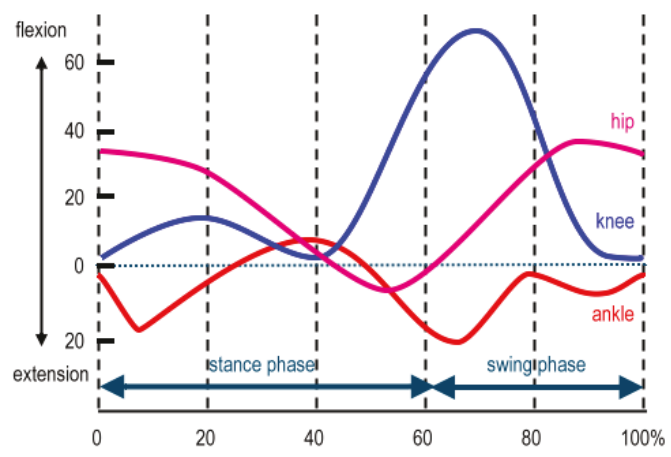

Fig. 3. Sagittal-plane motion of lower extremity during one gait cycle 
The trajectory of human walking was define using fifth order polynomials. These polynomials restrict the positions, velocities and accelerations and generate soft movements [7]. A path from $\theta_{\mathrm{i}}$ to $\theta_{\mathrm{f}}$ is defined as a continuous map, $\tau:[0,1] \rightarrow Q$, with $\tau(0)=\theta_{\mathrm{i}}$ and $\tau(1)=\theta_{\mathrm{f}}$. A trajectory is a function of time $\theta(t)$ such that $\theta\left(t_{0}\right)=\theta_{\mathrm{i}}$ and $\theta\left(t_{f}\right)=\theta_{\mathrm{f}}$. In this case, $t_{f}-t_{0}$ represents the amount of time taken to execute the trajectory. Since the trajectory is parameterized by time, we can compute velocities and accelerations along the trajectories by differentiation. If $\tau$ is time-dependent then a path is a special case of a trajectory, one that will be executed in one unit of time. In other words, in this case, $\tau$ gives a complete specification of the robot's trajectory, including the time derivatives (knowing the differentiate $\tau$, we can obtain time derivatives) [7].

\section{Design of the 3D System}

The proposed biped system has 6 degrees of freedom. The joints of the system are rotational. In the middle of the hip is located the base of the system, Fig. 4(a). The biped system is based on lower limb exoskeleton, Fig. 4(b). The system is drawn in SolidWorks ${ }^{\circledR}$.

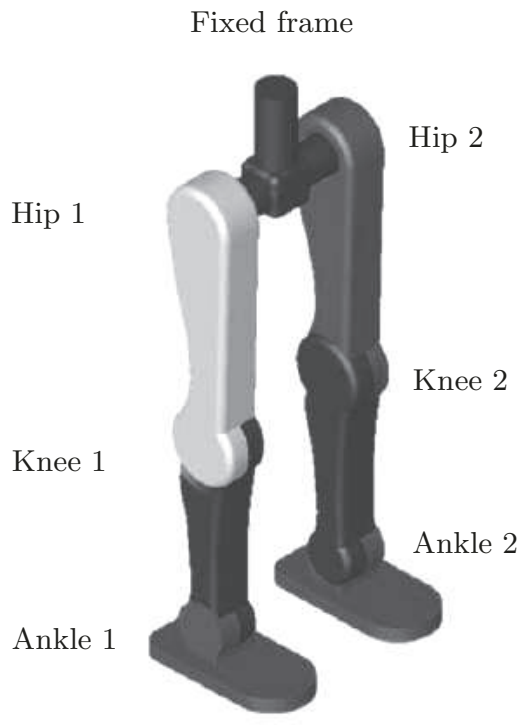

(a) SolidWorks ${ }^{\circledR}$ design

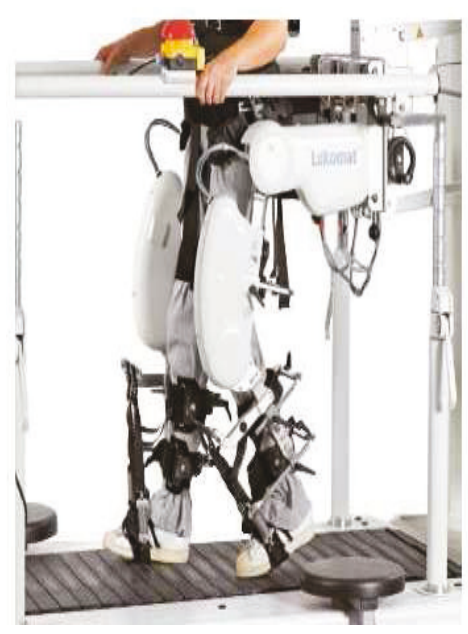

(b) Exoskeleton of lower limbs

Fig. 4. Biped system

Prismatic joints (translation movement) is one of the options to design a lower limb rehabilitation system. The main advantage of this technique is the simplicity 
in the modeling and implementation. However, designing a biped system considering rotational joints has the advantage of better leg kinematic description [8].

SolidWorks ${ }^{\circledR}$ allows us to applying the finite element analysis and define the physical properties of each element. These information is needed for movement modeling. Matlab ${ }^{\circledR}$ combines the physical properties, the dynamic model (mathematical representation of the system) and specific libraries in a programming environment [9].

\section{Simulator Designing}

We need several elements in order to develop the simulator: the dynamic model which describes the system's response to internal or external stimuli, the control law which controls the actions of the system to perform the task, the 3D model that allows us to visualize the system movements, and the physical parameters which give the system its physical characteristics, as seen in Fig. 5. The programming environment is Matlab ${ }^{\circledR}$.

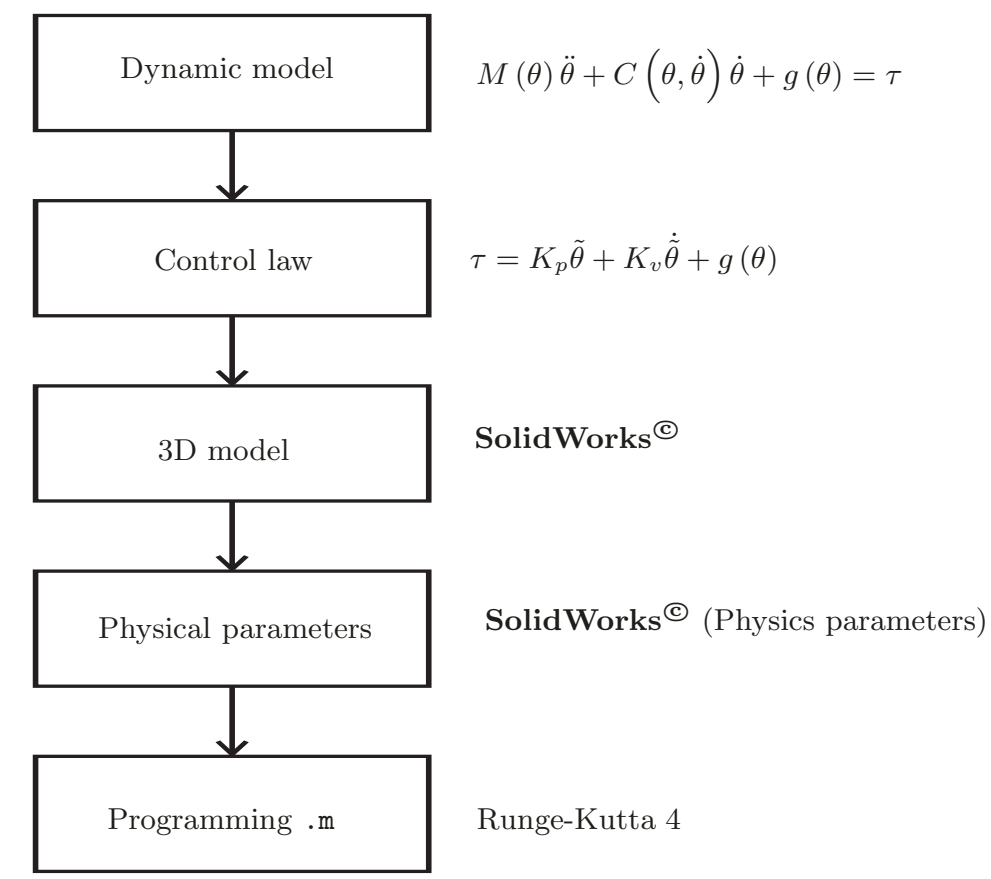

Fig. 5. Simulator elements

The programming language that set the numerical method is needed to relate these elements. Matlab ${ }^{\complement}$-Simulink uses Runge-Kutta 4 (ode45). 
The simulator uses the following procedure:
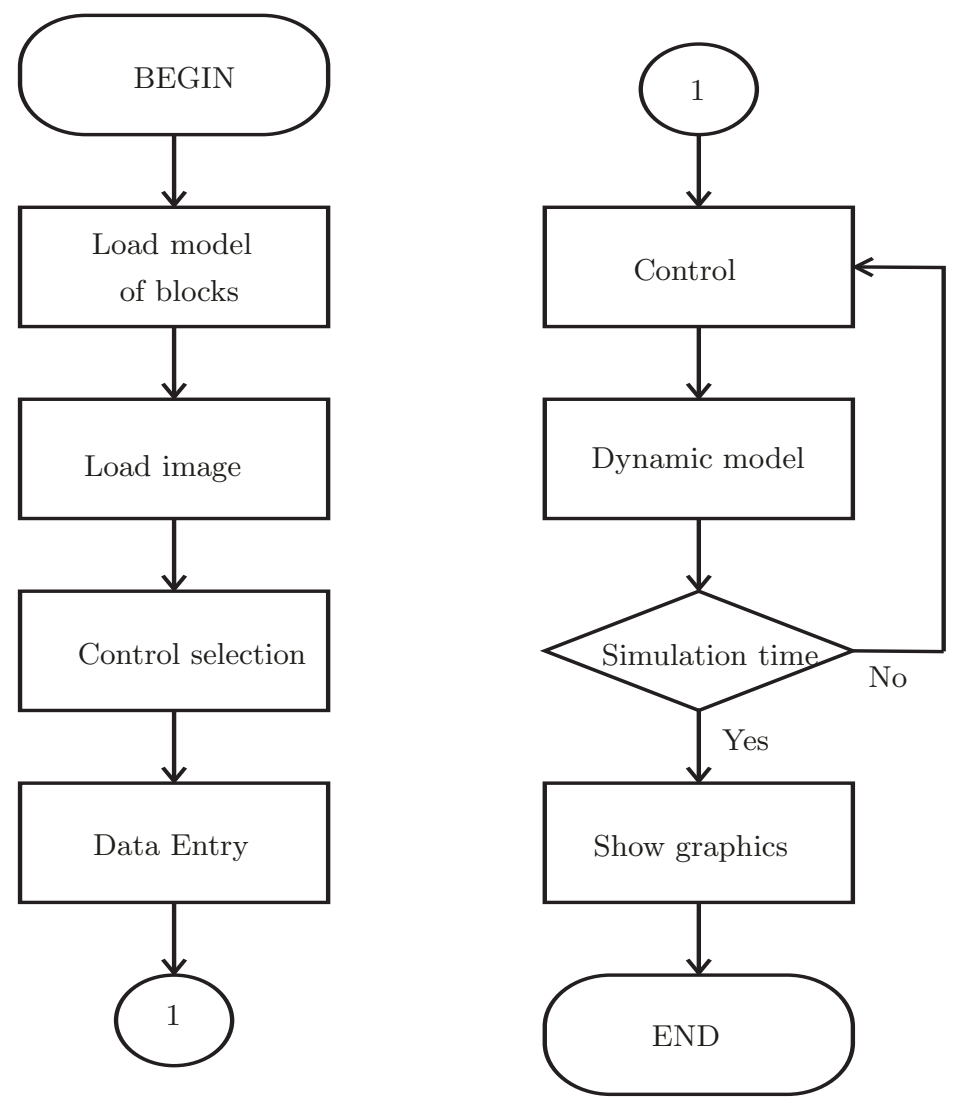

Fig. 6. Flow diagram

Matlab $^{\circledR}$ allows us to use the 3D image made in SolidWorks ${ }^{\circledR}$ using the Toolbox call as SimMechanics. This toolbox converts the image into an object, this object can moves according to the results of the numerical method ode 45 .

SimMechanics provides a multibody simulation environment for 3D mechanical systems. You model the multibody system using blocks representing bodies, joints, constraints, and force elements, and then SimMechanics formulates and solves the equations of motion for the complete mechanical system. Models from CAD systems requires mass, inertia, joint, constraint, and 3D geometry information. An automatically generated 3D animation lets us visualize the system dynamics. 


\section{Results}

Positions for each joint of the right leg $\{S P D\}$ is shown in Fig. 7

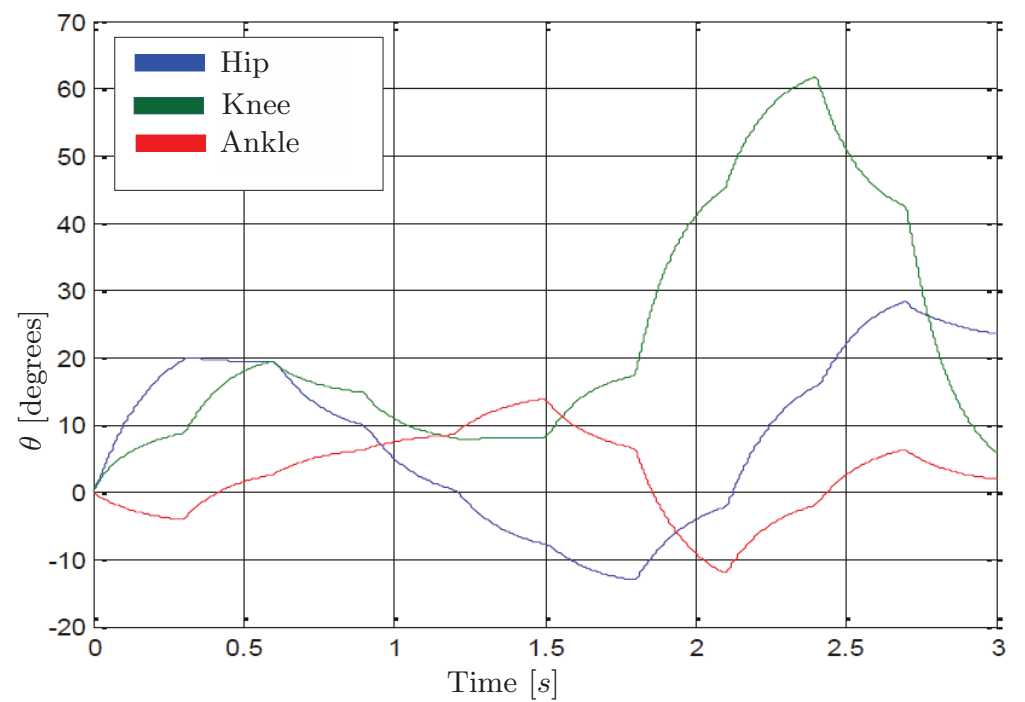

(a) Position

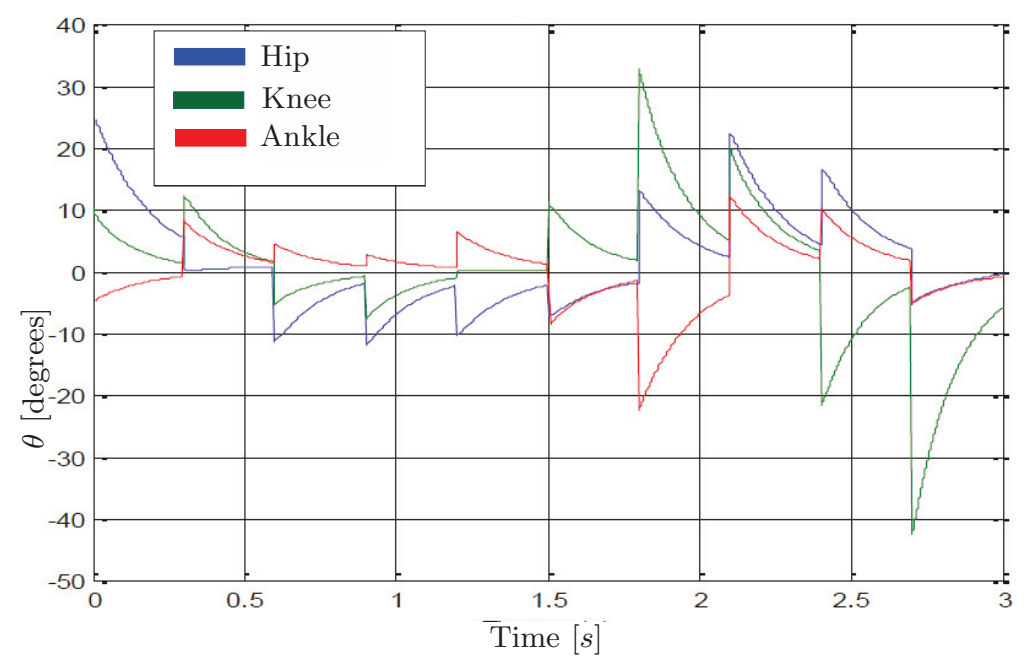

(b) Error position

Fig. 7. Position and error position results (right leg $\{S P D\}$ ) 
The trajectories obtained are similar to those shown in Fig. 3. To the left leg, the graphical output are similar but with a different start point to allow synchronization of the phases of support and oscillation of the movement of the legs in the running. Fig. 8 shows the human gait shown by the simulator.
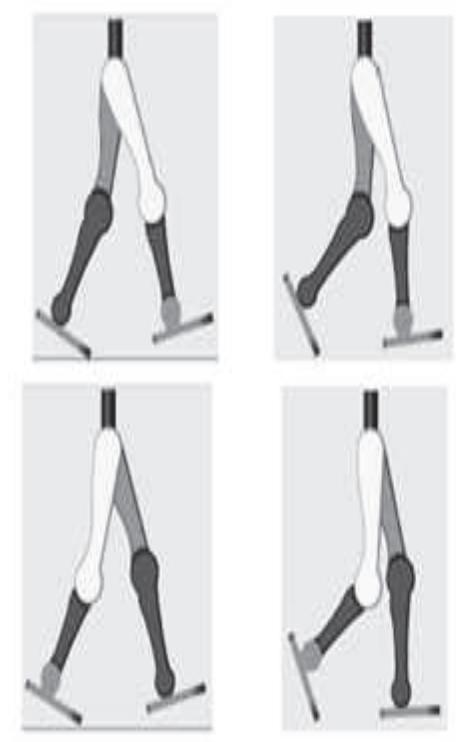
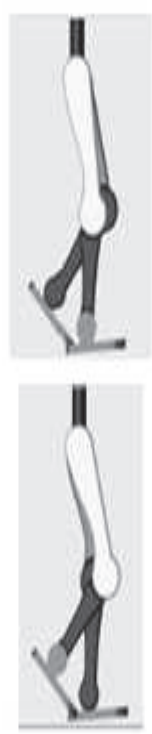
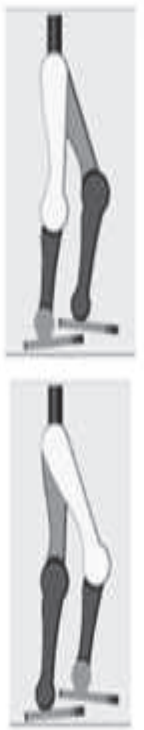
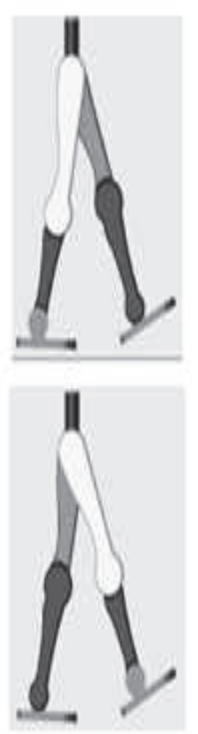

Fig. 8. Movement sequences

\section{Conclusion}

We developed a simulator using the system dynamic model, the system parameters, the implementation of a control law, the numerical method (RungeKutta), and the 3D drawing. The trajectory defined by the fifth-order polynomial adequately approaches the human gait. The trajectory can be improved by reducing the distance between the points that define it. The gain tuning of the control structure markedly contributes to the desired response and we can see its behavior as an amplifier with adjustable gain. The trajectory, defined by the fifth-order polynomial and the adequate selection of gains, allows the simulator efficiently replicate the human walking. Our results describe the system without interaction with the environment (contact with the floor, resistance to movement or other variables that depend on the time and velocity). The biped system analysis and the development of the simulator are the starting point for the development of a prototype with application in rehabilitation. 


\section{References}

1. Moore, H.: MATLAB for Engineers. Prentice Hall (2014)

2. The MathWorks, http://www.mathworks.es/discovery/robotica.html

3. Kajita, S. and Espiau, B.: Legged robots. In: Siliciano, B. and Khatib, O. (eds.) Handbook of Robotics 2008. pp. 361-387. Springer (2008)

4. Fu, K. S., González, R. C. and Lee, C. S. G.: Robótica: control, detección, visión e inteligencia. McGraw-Hill, USA (1987)

5. Kelly, R. and Santibáñez, V.: Control de movimiento de robots manipuladores. Prentice Hall, Madrid, España (2003)

6. Nordin, M. and Frankel, V. H.: Biomecánica básica del sistema musculoesquelético. McGraw-Hill, España (2004)

7. Spong, M. W., Hutchinson, S., and Vidyasagar, M. : Robot Modeling and Control. John Wiley and Sons Inc., New York (2007)

8. Van der Loos, M. and Reinkensmeyer, D. J.: Reahilitation and health care robotics. In: Siliciano, B. and Khatib, O. (eds.) Handbook of Robotics 2008. pp. 1223-1246. Springer (2008)

9. Valdivia, C. H. G., Ortega, A. B., Salazar, M. A. O., Rivera, H. R. A.: Modelado y simulación de un robot terapéutico para la rehabilitación de miembros inferiores. Revista Ingeniería Biomédica, Vol. 7, No. 14, pp. 42-50 (2013).

10. The MathWorks: SimMechanics User's Guide, http://www .mathworks .com

11. Ollero Baturone, A.: Manipuladores robóticos y robots móviles. Alfaomega, España (2007)

12. Ma, Y., Hei, W. and Sam Ge, S.: Modeling and control of a Lower-Limb rehabilitation robot. ICSR 2012, Springer, pp. 581-590 (2012). 